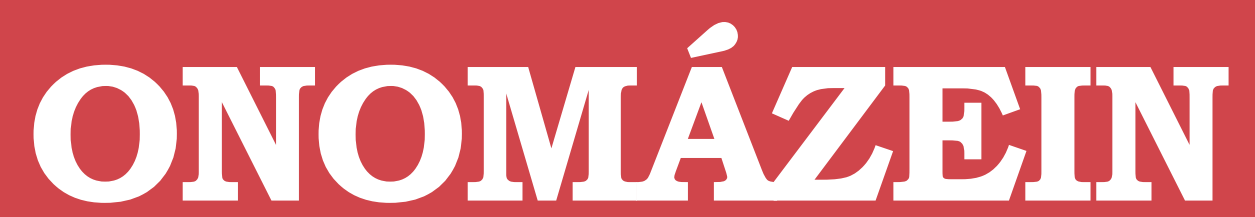

Revista semestral de lingüística, filología y traducción
PONTIFICIA UNIVERSIDAD CATÓLICA DE CHILE CATOLICA DE CHILE
FACULTAD DE LETRAS

\title{
El papel del alineamiento y la interacción comunicativa en la evolución de la capacidad humana para el lenguaje ${ }^{1}$
}

The role of alignment and communicative interaction in the evolution of the human capacity for language

Lucía Castillo Iglesias

Universidad de Chile Chile
Guillermo Soto Vergara

Universidad de Chile

Chile

Lucía Castillo Iglesias: Centro de Estudios Cognitivos, Facultad de Filosofía y Humanidades, Universidad de Chile. Chile. Correo electrónico: Iucia.castillo.iglesias@gmail.com

Guillermo Soto Vergara: Centro de Estudios Cognitivos, Departamento de Lingüística, Facultad de Filosofía y Humanidades, Universidad de Chile. Chile. Correo electrónico: guillermosotovergara@gmail.com 


\section{Resumen}

Contra la idea de que la capacidad para el lenguaje obedece a la evolución de un rasgo aislado, se propone que esta deriva de un conjunto de modificaciones asociadas a la evolución de un modo de vida prosocial en los homínidos. En particular, proponemos que el surgimiento de la gramática obedece a la estabilización de rasgos seleccionados mediante la interacción, cuya reiteración y sistematicidad derivan en la estandarización de comportamientos comunicativamente eficaces. La regularidad en el uso de estos elementos permite la emergencia cultural de la gramática como sistema de reglas que externaliza la estabilización. El alineamiento que se produce en la interacción comunicativa desempeña un papel crucial en este proceso.

Palabras clave: interacción; diálogo; alineamiento; evolución; gramática.

\section{Abstract}

Against the idea that the capacity for language evolved as an isolated feature, we propose that it derives from a set of modifications associated with the evolution of a prosocial way of life in hominids. In particular, we propose that the emergence of grammar results from the stabilization of features selected through interac- tion, whose reiteration and systematicity derive in the standardization of communicatively effective behaviors. Regularity in the use of these elements allows for the cultural emergence of grammar as a system of rules that externalizes stabilization. Interactive alignment plays a crucial role in this process.

Keywords: interaction; dialogue; alignment; evolution; grammar. 


\section{Introducción}

Confinado tradicionalmente al terreno de la especulación e incluso explícitamente excluido de la investigación lingüística², el estudio de los factores que inciden en la evolución de la facultad humana para el lenguaje ha experimentado, especialmente desde la aparición de "Natural language and natural selection" de Pinker y Bloom en 1990, un notable desarrollo, asociado a los avances que, en el mismo período, han tenido la ciencia cognitiva y las neurociencias (Soto y García, 1997; Christiansen y Kirby, 2003). Paradójicamente, mientras la propuesta de Pinker y Bloom buscaba integrar la lingüística generativa - específicamente la desarrollada en el marco de la teoría de principios y parámetros - con el evolucionismo darwiniano, la transformación que ha significado para las ciencias cognitivas la incorporación de las variables relativas a la posición del sujeto cognoscente en el mundo -asociadas a las hipótesis de cognición corporeizada, situada y distribuida (Clark, 1998; Anderson, 2003) - ha propiciado el desarrollo de posturas que cuestionan la aplicabilidad del enfoque lingüístico generativo tradicional en contextos evolutivos realistas. Estas nuevas posiciones se desarrollan en torno no ya de la supuesta génesis de un sistema formal de reglas en la mente individual, sino del papel que desempeñan la interacción y la comunicación en el surgimiento del lenguaje en la especie3, asumiéndolo como parte de un complejo proceso de transformaciones biológicas y culturales en el modo de vida de los homínidos que no puede explicarse únicamente apelando a la selección de adaptaciones específicas, como planteaban Pinker y Bloom .

La complejidad del problema - que aborda un periodo de la evolución humana del que se tienen datos limitados, y que pretende establecer criterios de demarcación en un proceso de huellas poco claras - hace necesario distinguir dos ámbitos de indagación relacionados: el de la "evolución de la capacidad para el lenguaje", asociado al estudio del paso filogenético de un estadio prelingüístico a uno lingüístico, y el de la "evolución de las lenguas", o glosogenia, referido a los procesos de transformación y transmisión cultural de las lenguas en sus comunidades hablantes. Si bien ambos convergen en el primer periodo lingüístico de la especie, esta distinción permite separar la investigación que aborda un problema evolutivo - el origen de la capacidad innata para aprender y utilizar una lengua, con las modificaciones morfológicas y cognitivas que ello implica (encefalización, descenso de la laringe, entre otras) - de otro cuyo análisis se centra en transformaciones exclusivamente culturales, como los cambios de la lengua en uso, la transmisión de la lengua de una comunidad generación tras generación, y las diferencias culturales que determinan modos de uso pragmático, reglas de socialización y cambio léxico, entre otros fenómenos.

Seguramente el aspecto más controversial en el estudio de la evolución del lenguaje radica en el interés de ambos campos de investigación -el que apela a la evolución biológica y el que lo hace a la cultura y la socialización- por explicar el surgimiento de la gramática. Aunque procesos como la gramaticalización ocurren en la escala de los fenómenos culturales (Hopper y Traugott, 1993), se han propuesto ya una gramática universal, ya universales lingüísticos de raíz biológica (Chomsky, 2000; Anderson y Lightfoot, 2002) que explicarían tanto estos fenómenos como la robustez de la adquisición del lenguaje

2 Prueba de ello son las, frecuentemente citadas, prohibiciones de abordar el tema formuladas por la Sociedad Lingüística de París, en 1866, y la Sociedad Filológica de Londres, en 1911 (Nerlich, 1989; Malmberg, 1990; Danesi, 1995).

3 Estas perspectivas presentan importantes similitudes con la propuesta de Maturana, Mpodozis y Letelier (1995), quienes destacan que el lenguaje surge en el dominio relacional como un modo de vida, y no en el cerebro como producto de las dinámicas del sistema nervioso.

4 Y otros, como Bickerton (1990). Una revisión crítica de estos enfoques en Soto y García(1997), Sampson (2005) y Aguilera (2007). 
en la especie. El problema surge cuando consideramos las diferencias entre quienes afirman que los universales han surgido como adaptaciones específicas para cierta facultad gramatical, cognitivamente encapsulada e innata en la especie, y quienes asumen que las lenguas que existen se han adaptado a las características generales de la cognición humana (la tesis de language as shaped by the brain de Christiansen y Chater, 2008). En términos globales, estas posiciones podrían resumirse en un gradiente de prioridad total del lenguaje, en el extremo generativo tradicional, versus prioridad total de la cognición general.

En los últimos años, esta polémica ha sufrido un nuevo vuelco, al ponerse en tela de juicio la existencia de universales lingüísticos específicos, los que no serían más que la expresión de constreñimientos generales derivados tanto de la naturaleza de la cognición humana y de procesos histórico-culturales (Evans y Levinson, 2009) como de la interacción de sucesivas generaciones de agentes (Kirby, 2000; Kirby y otros, 2007). Pretendemos, en este trabajo, mostrar ciertas debilidades de las propuestas evolutivas del generativismo tradicional y el funcionalista5, y delinear un escenario que considere los procesos biológicos, comunicativos y culturales que habrían dado origen a la comunicación lingüística humana.

\section{Posiciones en el debate acerca de la evolución de la facultad del lenguaje}

\subsection{El generativismo tradicional}

Contra quienes afirman que la interacción y la función comunicativa desempeñan un papel constitutivo en el sistema lingüístico, el generativismo tradicional, centrándose en el código y restringiéndose a representaciones y procesos individuales, sostiene que "el lenguaje es concebible como un sistema autónomo que puede analizarse productivamente sin considerar la dimensión social y comunicativa" (Soto, 2001: 116). Si bien muchos de sus autores aceptan que la comunicación incide diacrónicamente en la estructura lingüística, sostienen que el análisis puede limitarse al conocimiento lingüístico del hablante (la competencia) con independencia de su empleo en las situaciones comunicativas (la actuación), toda vez que la gramática se concibe como un sistema formal abstracto independiente del uso (Newmeyer, 2003).

Un problema fundamental de este enfoque es el de la adquisición de la lengua: el niño debe adquirir un complejo sistema de reglas a partir de un input por definición pobre. La complejidad de la tarea y las limitadas capacidades de aprendizaje del niño predecirían que muchos de ellos no adquirirían su lengua materna. Como este no es el caso, la única respuesta plausible es la preexistencia del conocimiento de la gramática de las lenguas, que se hallaría codificado genéticamente y se expresaría en la ontogenia ${ }^{6}$ en contacto con un entorno lingüístico determinado (Anderson y Lightfoot, 2002). Resulta esperable, en esta lógica, que el objeto de estudio evolutivo sea un "módulo del lenguaje", una gramática universal innata o un "dispositivo" que permita al niño adquirir la lengua de su comunidad (Chomsky, 1989). Como han afirmado Bates y Carnevale (1993), la fuerza del argumento depende de tres supuestos discutibles: el lenguaje corresponde a oraciones bien formadas generadas por un sistema gramatical formal abstracto ("a language is a state of the faculty of language", en palabras de Chomsky, 2005); el input es necesariamente

5 Optamos por estas denominaciones para referirnos, con la primera, a aspectos básicos del programa generativista, tal y como este se ha desarrollado hasta la teoría de principios y parámetros, y con la segunda, a la corriente que propone vincular el programa generativista con una versión fuerte del evolucionismo darwiniano. "Funcionalista", en consecuencia, no se emplea aquí ni en el sentido que tiene en lingüística ni en el que posee en teoría de la mente. La detención en ambas posiciones obedece al peso que han tenido en la discusión contemporánea sobre la relación entre mente, lenguaje y evolución.

6 La ontogenia corresponde al periodo de desarrollo de un organismo, en oposición a la filogenia, correspondiente a la historia evolutiva de la población o especie. 
pobre, y las capacidades de aprendizaje del niño se restringen a un modelo de verificación de hipótesis. Los tres supuestos han sido, total o parcialmente, puestos en tela de juicio por diversos autores, tanto teórica como empíricamente (Bates y Carnevale, 1993; Sampson, 2005; Lee y otros, 2009).

Con respecto a la evolución de la facultad lingüística, posiciones generativistas han sostenido que hay rasgos esenciales del lenguaje cuya evolución no es producto de un proceso gradual, sino de mutaciones unitarias y completas (Bickerton, 1990). Sin embargo, la idea de que el lenguaje es un sistema innato biológicamente complejo originado por una única mutación (un evento que "reorganizó el cerebro", según Chomsky, 2005) es difícil de sostener, toda vez que las mutaciones genéticas operan sobre unidades simples cuyo efecto individual - si bien pueden incidir en distintos rasgos en el fenotipo ${ }^{7}-$ no suele producir, por sí mismo, fenómenos de esta complejidad (Pinker y Bloom, 1990; Sampson, 2005; Dar-Nimrod y Heine, 2011) $)^{8}$.

En los últimos años, cierta corriente del generativismo, el denominado minimismo, ha acotado fuertemente el alcance de sus postulados, remitiendo aquellos aspectos no exclusivamente lingüísticos de la facultad del lenguaje al llamado "tercer factor", asociado a capacidades computacionales generales no especificadas (Chomsky, 2005), relacionadas, fundamentalmente, con la arquitectura estructural y las condiciones de eficiencia computacional. No obstante, el eje ha seguido estando puesto, en gran medida, en la aparición de una mutación súbita de efectos trascendentes (el "genotipo del lenguaje”), que se habría mantenido invariable en la especie desde su aparición hace 100 mil años y cuya función comunicativa sería secundaria a la de "instrumento del pensamiento" (Berwick y otros, 2013). Interesantemente, el creciente peso del denominado tercer factor ha tendido a asociarse a una reducción, a veces drástica (cfr. Hauser, Chomsky y Fitch, 2002; Berwick y otros, 2013), de la hipotetizada gramática universal y, consecuentemente, a un menor peso de argumentos característicos del generativismo tradicional, como el de la pobreza del input. En la medida en que, en esta orientación, el peso de gramática universal se reduce - eventualmente, hasta desaparecer-, el generativismo adquiere, por las razones ya expuestas, más plausibilidad evolutiva, otorga más relevancia a los procesos epigenéticos's, y puede, consecuentemente, ser compatible con aproximaciones emergentistas. Con todo, en tanto se mantiene el compromiso individualista (Berwick y otros, 2013; Chomsky, $2005)^{10}$, sigue siendo distinto de los enfoques que ubican el lenguaje en el espacio interactivo y que apuntan a la evolución cultural como un factor de cambio relevante.

\subsection{El generativismo funcionalista}

Si bien el generativismo funcionalista, tal como se presenta en Pinker y Bloom (1990), comparte con el tradicional la tesis de que el lenguaje es un sistema modular en el que las relaciones entre los elementos están predeterminadas y el uso solo presenta datos que activan mecanis-

7 El fenotipo corresponde a las características observables de un organismo, determinadas por la relación entre sus genes y el ambiente (Barton y otros, 2007). Más específicamente, designa al conjunto de tipos de rasgos posibles que un tipo génico o genotipo puede presentar (Mahner y Kary, 1997).

8 El sesgo derivado de la apelación al determinismo absoluto de los genes sobre rasgos morfológicos o conductuales ha sido denominado "genetic essentialism" (Dar-Nimrod y Heine, 2011), un problema que suele esconder la naturaleza compleja e interdependiente de los procesos evolutivos.

9 Los procesos epigenéticos corresponden a las diferencias en el desarrollo de los organismos que determinan la expresión diferencial de sus genes, contribuyendo a la manifestación de diferencias individuales (Jablonka y Lamb, 2005).

10 Chomsky es claro respecto al compromiso individualista y mentalista de esta perspectiva: "The biolinguistic perspective views a person's language as a state of some component of the mind, understanding 'mind' in the sense of eighteenthcentury scientists who recognized that after Newton's demolition of the only coherent concept of body, we can only regard aspects of the world 'termed mental' as the result of 'such an organical structure as that of the brain'” (2005: 2). 
mos innatos (Soto y García, 1997), difiere de este en su consideración de aspectos funcionales y evolutivos. En este enfoque, el lenguaje se define como un sistema complejo cuya función es comunicar proposiciones a través de una interfaz serial. La gramática, la fonética, la semántica y otros componentes habrían sido seleccionados para cubrir requerimientos específicos del sistema y se encontrarían codificados, en sus aspectos fundamentales, en el genoma humano. Más específicamente, se sostiene que la aparición del lenguaje es un fenómeno gradual explicable por presiones selectivas asociadas a su eficacia como herramienta de comunicación social (Pinker y Bloom, 1990) ${ }^{11}$.

El generativismo funcionalista hereda muchas de las críticas al sistema que afectan al tradicional, particularmente respecto a la codificación genética de la capacidad para el lenguaje y la gramática universal. Por otro lado, si bien su propuesta evolutiva es plausible, no es fácil de conciliar con datos como la gran diversidad lingüística humana; la dificultad para identificar universales propiamente lingüísticos; el papel de mecanismos cognitivos generales en el aprendizaje del lenguaje, y la importancia de mecanis mos de transmisión cultural en la conducta y el conocimiento del lenguaje (Kuhl, 2000; Jablonka y Lamb, 2005; Sampson, 2005; Evans y Levinson, 2009). La tesis del generativismo funcionalista descansa en la idea de que toda conducta compleja adaptativa robusta en la especie debe explicarse por selección natural, minimizando la importancia de otros procesos biológicos y de los mecanismos de transmisión social (Jablonka y Lamb, 2005); sin embargo, que el lenguaje constituya una propiedad universal de la especie, adecuada a su modo de vida, no determina la ne- cesidad de una explicación adaptacionista para su surgimiento (Kirby, 1998), sobre todo a la luz del rol que juegan en su estabilización los procesos culturales que derivan de su uso en interacción social, y que forman parte de la naturaleza misma del fenómeno.

\section{La cultura y el carácter social como variables de transformación: modelos co- evolutivos}

La gran variabilidad en la expresión "fenotípica" del lenguaje sugiere que los factores culturales y sociales desempeñan un papel decisivo en la emergencia y configuración de los sistemas lingüísticos (Bates, 1994; Sampson, 2005)². Desde una perspectiva que inserta la evolución del lenguaje en un marco cognitivo, social e interaccional amplio, la clave en el proceso evolutivo del cual surge el lenguaje simbólico no estaría en la aparición, súbita o progresiva, de un sistema especializado en procesar símbolos abstractos, sino en la selección del conjunto de habilidades sociales, cognitivas y emocionales que sustentan las complejas relaciones sociales características del modo de vida humano (Herrmann y otros, 2007) ${ }^{13}$. Entre estas, destacan las habilidades y motivaciones para la intencionalidad compartida, que serían fundamentales en el establecimiento de la "infraestructura cooperativa" que permite el aprendizaje y uso de símbolos (Tomasello y otros, 2005; Tomasello, 2008). En este escenario de comunicación prelingüística, los símbolos lingüísticos se incorporan progresivamente como "marcadores" a una rutina de uso de referencias compartidas en una comunidad, cargada de intencionalidad comunicativa desde su origen (Verhagen, 2005). Este proceso se explica a partir del carácter ostensivo-inferencial de

11 Esta definición adaptacionista se asocia a la Escuela de Santa Bárbara en psicología evolucionaria, que plantea que el lenguaje surgiría de la interacción entre evolución biológica y aprendizaje individual, minimizando la incidencia de la transmisión cultural.

12 Como muestran las lenguas de señas, la variación no se da solo en la modalidad audio-oral.

13 Diversos estudios muestran que el dominio del mundo físico y la inteligencia general de primates no humanos y niños pequeños son semejantes. La diferencia entre ambos grupos radica en que los niños emplean complejas habilidades sociales no observadas en primates no humanos (Herrmann y otros, 2007). 
la comunicación humana, centrada no solo en la codificación y decodificación de signos sino en la intención, por parte del hablante, de comunicar públicamente cierto contenido que modifique los supuestos cognitivos del interlocutor, y en el reconocimiento inferencial, por parte del oyente, de la intención comunicativa y social del hablante (Sperber y Wilson, 1995 [1986], 2002; Tomasello, 2008; Scott-Phillips, 2010).

En esta perspectiva, las modificaciones biológicas asociadas a la evolución de la capacidad para el lenguaje - en particular las referidas al procesamiento cognitivo - se orientan al establecimiento de mecanismos flexibles, abiertos a la reconfiguración adaptativa en el individuo y vinculados fundamentalmente a procesos hebbianos relativos a la frecuencia de uso, y no al de propiedades estructurales universales (Steels, 2008), como se explicará en la siguiente sección. Muchos rasgos en apariencia comunes a todas las lenguas parecen deberse a propiedades de otras capacidades cognitivas, como memoria, control motor, integración sensorial, etc.; a constreñimientos generales a los sistemas de comunicación, como la necesidad de distinguir entre elementos funcionales "cerrados" y "abiertos"; o a necesidades generales de la comunicación social humana, independientes del lenguaje (Evans y Levinson, 2009). A estos factores, se agregan las constantes sensorio-motoras que, actuando como elemento estable en nuestra configuración del mundo y asociadas a nuestra posición ecológica, imponen exigencias a todo sistema de comunicación (Tomasello, 2008). Finalmente, la evolución cultural de las lenguas también juega un papel determinante, en tanto permite la emergencia de estructuras lingüísticas a par- tir de su adaptación funcional a los objetivos comunicativos del individuo/población (Kirby, 1998; Steels, 2011) 14.

La enorme diversidad y variabilidad de las lenguas ${ }^{15}$ tendría su origen, por su parte, en la diversificación y acumulación de variación al interior de los grupos culturales, en un proceso similar al de la acumulación de variación genética por comunidades aisladas (Kirby, 1998; Evans y Levinson, 2009). La capacidad de adaptación cultural de los grupos, que puede aumentar drásticamente la variación conductual y sostener en el tiempo rasgos que difícilmente habrían sido seleccionados mediando únicamente la selección natural (Smith y Kirby, 2008; Smith, 2011), se asocia además a la búsqueda de mecanismos de identificación y diferenciación de grupos ${ }^{16}$. La pragmática asociada al uso del lenguaje simbólico reflejaría prácticas sociales implicadas en la coordinación de perspectivas necesaria para la comunicación efectiva (Brooks y Ragir, 2008). En este sentido, el aprendizaje de la lengua de la comunidad se vuelve un mecanismo de articulación social y de inclusión del niño en la relación con el mundo que esa comunidad ha plasmado en su uso de la lengua. La adquisición de la lengua por parte del niño no se reduce a la "activación" de mecanismos predeterminados genéticamente, sino que obedece a un aprendizaje social en contextos interaccionales en que el uso del lenguaje que se aprende es, a la vez, el medio de aprendizaje (Steels, 2004; Brighton y otros, 2005; Lee y otros, 2009) ${ }^{17}$. No existe, en este proceso, una lengua ideal que aparezca como referente abstracto al niño que aprende ni tampoco un sistema de reglas innato que deba activarse, sino una pluralidad de interacciones dife-

14 Esto implica que el funcionalismo aparece como principio rector no ya de la evolución biológica, sino de la adaptación cultural de un sistema orientado por el modo de vida comunicativo de sus usuarios.

15 Habría en la actualidad entre 5000 y 8000 lenguas (Evans y Levinson, 2009).

16 La maximización de las diferencias intergrupales y las semejanzas intragrupales sería la clave del mecanismo evolutivo de selección de grupo cultural (Boyd y Richerson, 2005, 2006, entre otros).

17 La repetición de este proceso a lo largo de muchas generaciones impactaría en las estructuras de las lenguas, que se "adaptarían" a esta lógica de transmisión. El modelamiento computacional de este fenómeno se conoce como "aprendizaje iterativo" (Kirby y Hurford, 2002, Brighton y otros, 2005, entre otros). 
rentes (Kirby, 1998) que permiten, una vez que su sistematicidad ha asegurado un piso mínimo de estabilidad, la abstracción de un sistema de producción lingüística creativo y pragmáticamente adecuado (Steels, 2004).

\section{El alineamiento y el surgimiento de la gramática}

En el marco hasta aquí expuesto, se ha planteado que la interacción lingüística cara a cara constituye no solo el escenario evidente del desarrollo ontogenético del lenguaje, sino también un proceso cuya reiteración genera las regularidades que posibilitan la estabilización de las lenguas como sistemas en el tiempo (Kirby, 1998, 2000; Levinson, 2006; Lee y otros, 2009). La importancia de la interacción dialógica como mecanismo de coordinación de contenidos y formas de comunicación ha sido destacada por distintos autores (Clark y Wilkes-Gibbs, 1986; Garrod y Pickering, 2004; Verhagen, 2005). Garrod y Pickering (2004) han propuesto que el procesamiento interactivo genera un "alineamiento conversacional" simultáneo e inconsciente de las representaciones lingüísticas de los interlocutores que, operando en todos los niveles del lenguaje (fónico, léxico, sintáctico y discursivo), reduce la complejidad computacional de la tarea conversacional ${ }^{18}$. Estudios recientes sugieren que este alineamiento implica la sincronización de los procesos neuronales asociados (Stephens y otros, 2010). Más ampliamente, como apuntan los propios Garrod y Pickering, el proceso se relaciona con vínculos entre la percepción y la acción, extensamente distribuidos en nuestra configuración neural, que están en la base del aprendizaje de conductas sociales como, entre otros, el lenguaje o la danza (Kuhl, 2000; Kirschner y Tomasello, 2009; Yun y otros, 2012). El alineamiento conversacional correspondería, así, a la incorporación de la comunicación lingüística a un proceso presente de manera extendida en nuestra interacción social, derivado, con toda probabilidad, de nuestras capacidades imitativas y de la prosocialidad que las explica. El alineamiento en sí mismo sería, según algunos autores, una forma de imitación que combina nuestras complejas redes de percepción-acción con un importante componente prosocial (Menenti y otros, 2012), cuyas formas básicas estarían ya presentes en la atención compartida que poseen también otras especies de primates (Carpenter y Liebal, 2011). El alineamiento, al igual que otras formas de imitación (como la imitación de gestos, posturas y manerismos, con las que suele coocurrir), promueve sentimientos de empatía y convergencia entre los interlocutores, y contribuye a la integración del grupo social (Chartrand y Bargh, 1999; Hecht y otros, 2012).

Proponemos que los procesos que permiten el alineamiento conversacional desempeñarían un papel crítico en la aparición y el desarrollo de las lenguas en grupos humanos con las propiedades cognitivas y sociales ya reseñadas. Por una parte, en virtud de la capacidad de los individuos de replicar neuronal y procedimentalmente el patrón que da origen a la secuencia motora que perciben (Stephens y otros, 2010), habrían posibilitado la estabilización, en los grupos, de secuencias de movimientos gestuales y orofaciales de gran complejidad, los que aportarían la infraestructura mecánica de la comunicación lingüística ${ }^{19}$. Al permitir, además, la coordinación de representaciones cognitivas en las interacciones comunicativas de los homínidos prelingüísticos, habrían hecho posible la estabilización de los patrones de interacción exitosos, lo que habría llevado, finalmente, al surgimiento

18 Mientras en el nivel discursivo los interlocutores seleccionan un marco de referencia común, en el sintáctico tienden a utilizar los mismos tipos de estructuras (incluso cuando se habla en dos lenguas distintas); en el léxico, a repetir las mismas expresiones de referencia para los mismos objetos (y a cambiarlas si el interlocutor las cambia); y en el fónico, a homogeneizar el tono fundamental (Quezada y otros, 2012) y la velocidad de articulación.

19 Como afirma, entre otros, Tomasello (2008), la evolución de la comunicación humana habría tenido un estadio inicial de articulación gestual intencional, seguido por uno de articulación orofacial. 
de convenciones comunicativas y, más específicamente, convenciones estrictamente lingüísticas; en otras palabras, lenguas humanas.

Nuestra propuesta supone que los procesos que permiten el alineamiento conversacional estaban ya presentes antes del surgimiento de las lenguas, en homínidos prelingüísticos que coordinaban sus representaciones cognitivas en la interacción comunicativa mediante el alineamiento de gestos y movimientos. La coordinación social de gestos y movimientos estaría presente también en otros primates, como permiten asumir los recientes resultados de Nagasaka y colaboradores (2013), en los que la sincronización motora de parejas de macacos rhesus muestra mayor efectividad y precisión en presencia directa del coparticipante, sugiriendo que las bases conductuales que permiten el alineamiento son anteriores al surgimiento del lenguaje y están presentes en la sincronización de conductas sociales en primates de manera amplia. El alineamiento habría favorecido la solución de problemas propios de la vida social que suponían la coordinación de las conductas de los actores y la integración de los grupos en torno a modos específicos de resolver problemas comunes, fundamental en la supervivencia y la evolución de nuestro linaje. La comunicación humana se desarrollaría, entonces, sobre una infraestructura de coordinación y sincronización conductual, representacional y neuronal, que habría permitido a los individuos comunicarse efectivamente mediante el establecimiento de referencias compartidas de manera multimodal, con anterioridad a la emergencia de un código lingüístico común.

En este contexto, la reiteración de los patrones de interacción comunicativa exitosos posibilitaría la estabilización de las asociaciones entre determinadas conductas comunicativas y sus significados en la interacción, posibilitando el surgimiento del lenguaje simbólico, en un proceso que ha sido replicado experimentalmente con éxito en la emergencia de sistemas gráficos de comunicación (Galantucci, 2005; Garrod y otros, 2007; Fay y otros, 2010; entre otros). La emergencia de formas convencionales depende, en estos escenarios experimentales, tanto del éxito comunicativo y la frecuencia de uso asociada como de las características intrínsecas del símbolo que ayudan a su memorización (sesgos de aprendizaje o de contenido) (Fay y otros, 2010)20.

Según Steels (2011), el alineamiento se genera en un sistema cibernético que evalúa automáticamente la eficacia comunicativa de las construcciones en uso, de manera que, en futuras interacciones, la posibilidad de cada construcción de ser utilizada se asocia a la evaluación de sus usos anteriores, mediante una estrategia de inhibición lateral de construcciones con funcionalidad similar. Así concebido, el proceso implica la incorporación de un elemento de autoorganización mediante retroalimentación positiva en el sistema comunicativo, en tanto el éxito en el uso de una convención estimula su utilización y el mayor uso predice una mayor eficacia comunicativa. El proceso de selección, aunque automático en su mecánica, estaría guiado por preferencias de naturaleza cognitivo-funcional que mejorarían las posibilidades de propagación de estas formas en la población: facilidad de memorización, mejor reconocimiento de límites y mejor comprensión (Steels, 2011). Este proceso permitiría la estabilización de convenciones comunicativas en el surgimiento de las lenguas y en su desarrollo histórico.

Dada esta constante evaluación -que podríamos asumir como la raíz tanto de la estabilización como de la variación en el lenguaje-, la sistematicidad de las lenguas puede ser entendida como un fenómeno que emerge del alineamiento distribuido en poblaciones en interac-

20 Kirby (2000) y Kirby y Hurford (2002), entre otros, han llegado a conclusiones semejantes. Mediante el modelamiento de sucesivas generaciones de agentes con vocabularios simples, la sintaxis emerge automáticamente a partir de la reiteración de las interacciones. 
ción comunicativa. La gramática correspondería, así, a un nivel de abstracción de estabilidades que permite la identificación, tanto automática como consciente, de las construcciones más utilizadas y, por tanto, la posibilidad de abstraer estas construcciones de sus contextos de uso y de entenderlas como sistemas de regularidades de mayor o menor alcance. En el plano de la mente individual, el proceso descansaría en capacidades cognitivas generales de comparación y abstracción de patrones, ampliamente reconocidas por la bibliografía psicológica contemporánea, que están en la base de las denominadas "gramáticas basadas en el uso" (Langacker, 1987; Bybee, 2006; entre otros). Un enfoque de este tipo es compatible con la noción de "gramaticalización" desarrollada por la lingüística contemporánea, que apunta a diversos procesos temporales en que la gramática surge de la sedimentación de patrones de uso frecuente que se convencionalizan en la medida en que son adoptados por la comunidad hablante (Hopper y Traugott, 1993; Bybee y otros, 1994; Evans y Levinson, 2009). Permite explicar, también, la degradación lingüística (language attrition) que sufren los sistemas gramaticales por su no empleo en instancias de interacción comunicativa.

Dado el contexto hasta aquí expuesto, sostenemos que el progresivo aumento del tamaño de los grupos humanos (Dunbar, 1993), asociado al incremento de las interacciones con desconocidos en nuevos contextos, habría acelerado el proceso de selección de estructuras estables, propiciando mayores niveles de estandarización ${ }^{21}$. Esto se debería a que las interacciones comunicativas irían crecientemente dependiendo más de las estructuras lingüísticas que de factores contextuales. La escritura, al eliminar las restricciones espacio-temporales en que se da la in- teracción comunicativa humana original, habría, con toda probabilidad, favorecido aún más este proceso. La estabilidad de las gramáticas permitiría, a su vez, que las lenguas pudieran ser tratadas como objetos en sí mismas, atribuyéndoles propiedades externas a la dinámica de la comunicación. Las gramáticas adquirirían, así, estatus de norma en las comunidades, tanto implícita como explícitamente, en un proceso probablemente potenciado no solo por la escritura sino también por los sistemas de educación formal.

\section{Conclusión}

En el presente trabajo hemos argumentado que el surgimiento del lenguaje y las lenguas humanas no obedece a un proceso evolutivo darwiniano específico ni resulta de una mutación singular o una exaptación²2. A nuestro juicio, el lenguaje y las lenguas surgen de un conjunto de transformaciones asociadas al modo de vida prosocial de nuestra especie, caracterizado por interacciones comunicativas cara a cara. En particular, el alineamiento que se produce, en distintos niveles, durante estas interacciones habría favorecido el desarrollo de las gramáticas, entendidas como sistemas de signos en uso y no como meras reglas abstractas. La reiteración de las interacciones habría favorecido la introyección de las gramáticas en el individuo y su estabilización en los grupos. Este último proceso, a su vez, habría sido potenciado por el crecimiento de los grupos humanos y el desarrollo y expansión de la escritura y la educación formal. En nuestra propuesta, las lenguas dejan de entenderse como sistemas encapsulados que existen ya en un individuo ideal, ya en un ente reificado - la sociedad - (Steels, 1999; Soto y Hasler, 2011) para ser comprendidas en la complejidad de la red de estructuración social en que los seres humanos habitamos comunicativamente.

21 Para Dezecache y Dunbar (2012), la risa sirvió como un mecanismo prelingüístico de cohesión social que habría aumentado el tamaño de los grupos, al multiplicar los vínculos que antes podían establecerse solo por grooming diádico.

22 Una exaptación corresponde a un rasgo que no fue seleccionado por selección natural para la función que desempeña en el momento de la observación (Vargas, 2005), habiendo desempeñado, frecuentemente, una función previa que dejó de tener relevancia selectiva. 


\section{Bibliografía citada}

Aguilera, Bernardo, 2007: Origen y evolución de la facultad del lenguaje desde una perspectiva dinámica. Tesis de Magíster en Estudios Cognitivos, Universidad de Chile.

Anderson, Stephen y David Lightfoot, 2002: The Language Organ: Linguistics as Cognitive Physiology, Cambridge: Cambridge University Press.

Anderson, Michael, 2003: "Embodied Cognition: A field guide”, Artificial Intelligence 149, 91-130.

BARTON, Nicholas y otros, 2007: Evolution, Cold Spring Harbor, NY: Cold Spring Harbor Laboratory Press.

BATES, Elizabeth, 1994: "Modularity, domain specificity and the development of language", Discussions in Neuroscience 10, 136-149.

Bates, Elizabeth y George Carnevale, 1993: "New directions in research on language development", Developmental Review 13, 436-470.

Berwick, Robert, Angela Friedericl, Noam Chomsky y Johan Bolhuis, 2013: "Evolution, brain, and the nature of language", Trends in Cognitive Sciences 17, 89-98.

Bickerton, Derek, 1990: Language and Species, Chicago: University of Chicago Press.

Boyd, Robert y Peter Richerson, 2005: Not by Genes Alone: How culture transformed human evolution, Chicago: University of Chicago Press.

Boyd, Robert y Peter Richerson, 2006: "Culture and the Evolution of Human Social Instincts" en Stephen Levinson y Nick Enfield (eds.): Roots of Human Sociality, Berg: Oxford, 453-477.

Brighton, Henry, Kenny Smith y Simon KIRBY, 2005: "Language as an evolutionary system", Physics of Life Reviews 2, 177-226.

Brooks, Patricia y Sonia RagIR, 2008: "Prolonged plasticity: Necessary and sufficient for language- ready brains", Behavioral and Brain Sciences 31 , 514-515.

Bybee, Joan, 2006: "From usage to grammar: the mind's response to repetition", Language 82, 529551.

Bybee, Joan, Revere Perkins y William Pagliuca, 1994: The evolution of grammar: Tense, aspect and modality in the languages of the world, Chicago: The University of Chicago Press.

Carpenter, Melinda y Kristin Liebal, 2011: "Joint Attention, Communication, and Knowing Together in Infancy" en Axel Seemann (ed.): Joint attention: New developments in psychology, philosophy of mind, and social neuroscience, Cambridge, MA: MIT Press, 159-181.

Chartrand, Tanya L. y John A. Bargh, 1999: "The chameleon effect: The perception-behavior link and social interaction", Journal of Personality and Social Psychology 76 (6), 893-910.

Chomsky, Noam, 1989: "Cambios de perspectiva sobre el conocimiento y uso del lenguaje" en A. Alonso Cortés (ed.): Lecturas de lingüística, Madrid: Cátedra, 163-214.

Chomsky, Noam, 2000: New Horizons in the Study of Language and Mind, Cambridge: Cambridge University Press.

Сномsку, Noam, 2005: "Three factors in language design”, Linguistic Inquiry 36, 1-22.

Christiansen, Morten y Nick Chater, 2008: "Language as shaped by the brain", Behavioral and Brain Sciences 31, 489-558.

Christiansen, Morten y Simon Kirby, 2003: "Language evolution: consensus and controversies", Trends in Cognitive Sciences 7, 300-307.

Clark, Andy, 1998: Being there. Putting brain, body, and world together again, Cambridge, MA: MIT Press.

Clark, Herbert y Deanna Wilkes-GibBS, 1986: "Refer- 
ring as a collaborative process", Cognition 22, 1-39.

DanesI, Marcel, 1995: "The question of language origins in contemporary focus", Semiotica 103, 309-326.

Dar-Nimrod, Ilan y Steven Heine, 2011: "Genetic essentialism: On the deceptive determinism of DNA", Psychological Bulletin 137, 800-818.

Dezecache, Gillaume y Robin Dunbar, 2012: "Sharing the joke: the size of natural laughter groups", Evolution and Human Behavior 33, 775-779.

Dunbar, Robin, 1993: "Coevolution of neocortical size, group size and language in humans", Behavioral and Brain Sciences 16, 681-735.

Evans, Nicholas y Stephen Levinson, 2009: "The myth of language universals: Language diversity and its importance for cognitive science", Behavioral and Brain Sciences 32, 429-492.

Fay, Nicolas, Simon Garrod, Leo Roberts y Nik SwoBODA, 2010: "The interactive evolution of human communication systems”, Cognitive Science 34, 351-386.

Galantuccl, Bruno, 2005: "An experimental study of the emergence of human communication systems", Cognitive Science 29, 737-767.

Garrod, Simon y Martin Pickering, 2004: "Why is conversation so easy?", Trends in Cognitive Science 8, 8-11.

Garrod, Simon, Nicolas Fay, John Lee, Jon Oberlander y Tracy MACLEOD, 2007: "Foundations of representation: Where might graphical symbol systems come from?", Cognitive Science 31, 961-987.

Hauser, Marc D., Noam Chomsky y W. Tecumseh FITCH, 2002: "The faculty of language: what is it, who has it and how did it evolve?", Science 298, 1569-1579.
Hecht, Erin, Richard Patterson y Aron Barbey, 2012: "What can other animals tell us about human social cognition? An evolutionary perspective on reflective and reflexive processing", Frontiers in Human Neuroscience 6: 224.

Herrmann, Esther y otros, 2007: "Humans have evolved specialized skills of social cognition: The cultural intelligence hypothesis", Science 317, 1360-1366.

Hopper, Paul y Elizabeth Traugott, 1993: Grammaticalization, Cambridge: Cambridge University Press.

Jablonka, Eva y Marion LAmb, 2005: Evolution in Four Dimensions, Cambridge: The MIT Press.

KIRBY, Simon, 1998: "Fitness and the selective adaptation of language" en James Hurford, Michael Studdert-Kennedy y Chris Knight (eds.): Approaches to the Evolution of Language: Social and Cognitive Bases, Cambridge: Cambridge University Press, 359-383.

KIRBY, Simon, 2000: "Syntax without natural selection: How compositionality emerges from vocabulary in a population of learners" en Chris KNIGHT (ed.): The Evolutionary Emergence of Language: Social Function and the Origins of Linguistic Form, Cambridge: Cambridge University Press, 303-323.

KirBY, Simon y James Hurford, 2002: "The Emergence of Linguistic Structure: An overview of the Iterated Learning Model" en Angelo Cangelosi y Domenico ParIsI (eds.): Simulating the Evolution of Language, Londres: Springer Verlag, 121-148.

Kirby, Simon, Mike Dowman y Thomas Griffiths, 2007: "Innateness and culture in the evolution of language”, PNAS 104, 5241-5245.

Kirschner, Sebastian y Michael Tomasello, 2009: "Joint drumming: Social context facilitates synchronization in preschool children", Journal of Experimental Child Psychology 102, 299-314. 
Kunl, Patricia, 2000: "A new view of language acquisition”, PNAS 97, 11850-11857.

LANGACKER, Robert, 1987: Foundations of cognitive grammar: theoretical prerequisites, Stanford, CA: Stanford University Press.

LeE, Namhee y otros, 2009: The Interactional Instinct, Nueva York: Oxford University Press.

Levinson, Stephen, 2006: "On the human 'interaction engine"” en Nick ENField y Stephen Levinson (eds.): Roots of human sociality: Culture, cognition and interaction, Oxford: Berg, 39-68.

Mahner, Martin y Michael Kary, 1997: "What exactly are genomes, genotypes and phenotypes? And what about phenomes?", Journal of Theoretical Biology 186, 55-63.

Malmberg, Bertil, 1990: "Le débat sur l'origine du langage des encyclopédistes à Hermann Paul”, Studia Linguistica 44, 93-125.

Maturana, Humberto, Jorge Mpodozis y Juan Carlos Letelier, 1995: "Brain, Language and the Origin of Human Mental Functions", Biological Research 28, 15-26.

Menenti, Laura, Martin Pickering y Simon Garrod, 2012: "Towards a neural basis of interactive alignment in conversation", Frontiers in Human Neuroscience 6, 1-9.

Nagasaka, Yasuo y otros, 2013: "Spontaneous synchronization of arm motion between Japanese macaques", Scientific Reports 3, 1151.

Nerlich, Brigitte, 1989: "The evolution of the concept of 'linguistic evolution' in the 19th and 20th century", Lingua 77, 101-112.

Newmeyer, Frederick, 2003: "Grammar is grammar and usage is usage", Language 79, 682-707.

Pinker, Steven y Paul Bloom, 1990: "Natural Language and Natural Selection", Behavioral and Brain Sciences 13, 707-784.
Quezada, Camilo y otros, 2012: "Empatía y convergencia del tono fundamental", Revista de Lingüística Teórica y Aplicada 50, 145-165.

Sampson, Geoffrey, 2005: The "language instinct" debate, Londres: Continuum.

Scott-Phillips, Thomas, 2010: "The Evolution of Relevance”, Cognitive Science 34, 583-601.

Smith, Kenny y Simon KIRBY, 2008: "Cultural evolution: implications for understanding the human language faculty and its evolution", Philosophical Transactions of the Royal Society B 363, 35913603.

Smith, Kenny, 2011: "Learning Bias, Cultural Evolution of Language, and the Biological Evolution of the Language Faculty", Human Biology 83, 261278.

Sото, Guillermo, 2001: "Perspectivas para la lingüística: más allá de la dicotomía formalismo/ funcionalismo", Revista Chilena de Humanidades 21, 115-154.

Soto, Guillermo y Ricardo GARCíA, 1997: “Una visión del problema del origen del lenguaje en las ciencias cognitivas", Lenguas Modernas 24, 5-43.

Soto, Guillermo y Felipe Hasler, 2011: "Hacia una Dialectología cognitivo-funcional”, ponencia presentada en el III Simposio de la Asociación Argentina de Lingüística Cognitiva, Mar del Plata, Argentina.

Sperber, Dan y Deidre Wilson, 1995 [1986]: Relevance: Communication and Cognition, Oxford: Blackwell.

Sperber, Dan y Deidre Wilson, 2002: "Pragmatics, modularity and mind-reading", Mind \& Language $17,3-23$.

Steels, Luc, 1999: "The puzzle of language evolution”, Kognitionswissenschaft 8, 143-150.

Steels, Luc, 2004: "Social and Cultural Learning in the Evolution of Human Communication" en Kimbrough Oller y Ulrike Griebel (eds.): Evolution 
of communication systems: A comparative approach, Cambridge: Bradford Books, 69-89.

Steels, Luc, 2008: "Cognition and social dynamics play a major role in the formation of grammar" en Derek Bickerton y Eörs Szathmary (eds.): Biological Foundations and Origin of Syntax, Strüngmann Forum Reports, vol. 3, Cambridge, MA: MIT Press, 345-368.

Steels, Luc, 2011: "Modeling the cultural evolution of language", Physics of Life Reviews 8, 339-356.

Stephens, Greg, Lauren Silbert y Uri Hasson, 2010: "Speaker-listener neural coupling underlies successful communication”, PNAS 107, 14425-14430.

Tomasello, Michael y otros, 2005: "Understanding and sharing intentions: The origins of cultural cognition", Behavioral and Brain Sciences 28, 675-691.

Tomasello, Michael, 2008: Origins of Human Communication, Cambridge MA: The MIT Press.

VARGAS, Alexander O., 2005: "Beyond selection", Revista Chilena de Historia Natural 78, 739-752.

Verhagen, Arie, 2005: Constructions of Intersubjectivity, Nueva York: Oxford University Press.

Yun, Kiongsik, Katsumi Watanabe y Shinsuke Shimojo, 2012: Interpersonal body and neural synchronization as a marker of implicit social interaction, Scientific Reports 2, 959. 